\title{
Photovoltaic (PV) Power Output Prediction Using LSTM Based Deep Learning
}

\author{
Kwanho Kim*1' Donghun Lee ${ }^{2}$ \\ ${ }^{1}$ Dept. of Industrial Management and Engineering, Incheon National University, South Korea. \\ ${ }^{2}$ Dept. of Industrial Management and Engineering, Incheon National University, South Korea. \\ Correspondence Author: Kwanho Kim, Dept. of Industrial Management and Engineering, Incheon National University, South Korea. \\ E-mail: khokim@inu.ac.kr
}

Received date: 22 April 2019, Accepted date: 22 July 2019, Online date: 25 August 2019

Copyright: (c) 2019 Kwanho Kim, Donghun Lee.2019. This is an open-access article distributed under the terms of the Creative Commons Attribution License, which permits unrestricted use, distribution, and reproduction in any medium, provided the original author and source are credited.

\begin{abstract}
Background: The photovoltaic (PV) power output prediction become a very important research topic for overall planning of PV facility systems. In particular, accurate PV power output in a peak zone prediction is important for PV operators who profit by selling electricity obtained from a PV supply operation system. However, prediction of PV power output in a peak zone without meteorological information is considered as a challenging problem since meteorological factors are difficult to determine for a specific area and dynamic and undergo random changes both in a day and across days. Objective: Therefore, in this paper, we propose a long short-term memory (LSTM) based model. The proposed model is aimed at understanding the hidden sequence patterns that correlate historical PV power outputs in a peak zone and their preceding information for precise PV power output in a peak zone prediction. Results: The experiment results demonstrate that the proposed models show more than $10 \%$ better performance compared to the conventional models. Conclusion: LSTM based deep learning model is effective to predict PV power, and more sophisticated methods are needed to be developed for further improvements.
\end{abstract}

Keywords: Photovoltaic (PV). Power Output. Prediction. LSTM Based Deep Learning

\section{INTRODUCTION}

Mainly, to increase the profits made by selling electricity generated in PV operation, the prediction of PV power outputs in a peak zone is essential for PV operators. In general, they sell electricity obtained from PV operation either in day-ahead markets [1] or intraday markets at a given price [2,3]. As intraday renewable markets, in which the price is decided real-time, have become popular, PV operators try to sell when the costs of electricity are higher.

Since the PV operating system influences the price in intraday renewable markets, depending on the quantity of electricity production [4], the rate is increased when a large amount of electricity is produced, therefore, a more sophisticated prediction of the PV power output in a peak zone is necessary.

To define the range of PV power output in a peak zone, and its preceding information, the distribution and cumulative distribution of PV power output obtained in a day, as illustrated in Figure 1(a) and (b), respectively, are used. The peak zone is the times period in which the estimated PV power output during a day is highest, and its preceding information consists of the PV power outputs prior to the peak zone, and meteorological and seasonal information. There has been some research that is to predict PV power outputs using deep learning technique. DNN based models that consist of one or more hidden layers predict the PV power output using meteorological information [5] and clearness index [6]. For more sophisticated prediction for the PV power output, recurrent neural network (RNN) and long short term memory (LSTM) based models were proposed [7], using these, the PV power outputs are predicted after classifying weather data [8]. 


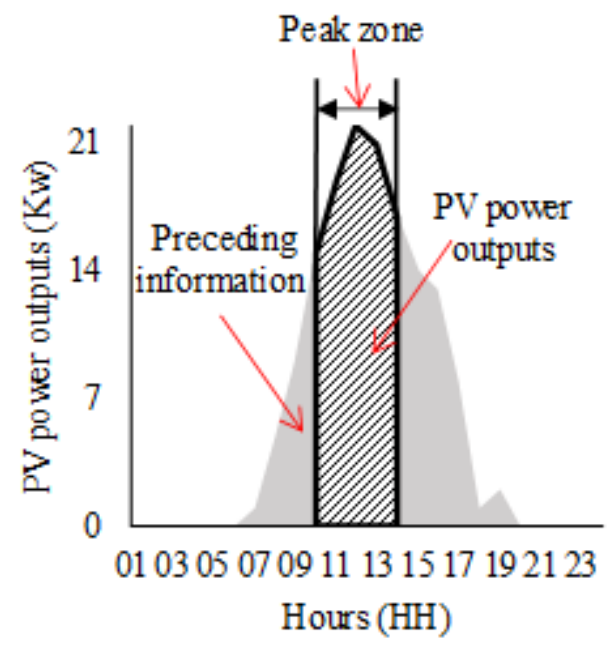

(a) Distribution of PV power outputs in a day (June 6,2015 )

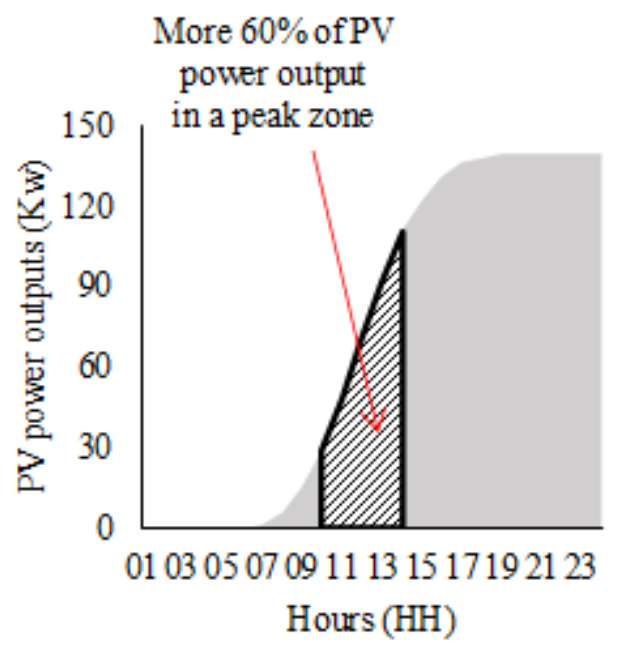

(b) Cumulative distribution of PV power outputs in a day (June 6,2015 )

Figure 1: Examples of PV power outputs distribution and cumulative distribution obtained in South Korea

Prediction of the PV power output in a peak zone without meteorological information is considered a challenging problem. It is well known that PV power output is affected by a variety of meteorological factors, such as solar radiation, temperature, and cloudiness. These factors are difficult to determine for a specific area because they are generally estimated for a wide range of geographic regions. Moreover, meteorological factors are dynamic and undergo random changes according to local weather conditions and seasonal patterns.

In this paper, we propose recurrent neural network-based models that succeed in predicting PV power output in the peak zone without using future meteorological information. The proposed models attempt to estimate the PV power output during the peak zone in a day by only utilizing the preceding information. Hence, our models can reliably predict he PV power outputs in the peak zone by eschewing future meteorological information, since accurate meteorological forecast information for a specific area is hard to determine.

The proposed LSTM models estimate the PV power outputs in a peak zone by sequentially utilizing the preceding information per hour without future meteorological information and other predicted PV power outputs. The experiment results of this study show that the proposed models successfully yield the PV power outputs in a peak zone by using a real-world dataset.

\section{PROPOSED METHOD}

In this study, we considered the problem of the estimating PV power outputs in the peak zone without future meteorological information. Table 1 describes the inputs and outputs with the notations used in this study. The inputs include preceding information such as meteorological, and seasonal data, and the PV power outputs before the peak zone. Four meteorological factors have consisted of temperature, humidity, cloudiness, radiation, and two seasonal factors, the month of the year and day of the month, were considered. The output includes only the PV power outputs in the peak zone.

Table 1: Input and output features considered

\begin{tabular}{|c|c|c|}
\hline & Notations & Descriptions $(k=1, \ldots, K, j=1, \ldots, J, d=1, \ldots, D)$ \\
\hline \multirow{7}{*}{ Inputs } & $t_{k, d}$ & The temperature of the k-th hour in the d-th day \\
\hline & $a_{k, d}$ & The humidity of the k-th hour in the d-th day \\
\hline & $c_{k, d}$ & Cloudiness of the k-th hour in the d-th day \\
\hline & \multirow{2}{*}{$\begin{array}{l}r_{k, d} \\
b_{k, d}\end{array}$} & Radiation of the k-th hour in the d-th day \\
\hline & & Day of month of the k-th hour in the d-th day \\
\hline & $\begin{array}{l}b_{k, d} \\
z_{k, d}\end{array}$ & Month of year of the k-th hour in the d-th day \\
\hline & $S_{k, d}$ & PV power output before the peak zone of the k-th hour in the d-th day \\
\hline
\end{tabular}




\begin{tabular}{|c|c|c|}
\hline $\begin{array}{c}\text { Predicted } \\
\text { Outputs }\end{array}$ & $\hat{y}_{j, d}$ & Predicted PV power output in the peak zone of the $\mathrm{j}$-th hour in the d-th day \\
\hline $\begin{array}{c}\text { Actual } \\
\text { Outputs }\end{array}$ & $y_{j, d}$ & Actual PV power output in the peak zone of the $\mathrm{j}$-th hour in the d-th day \\
\hline
\end{tabular}

Figure 2 shows the difference between an earlier model and the proposed model in terms of training and testing phases. The framework from the earlier study learns with future meteorological information, as shown in Figure 2 (a), whereas our framework learns without this information by assuming that the future meteorological information is unknown, as shown in Figure 2 (b). Therefore, our models evaluated the performance by calculating the difference between the predicted PV power in the peak zone $\hat{y}_{j, d}$ and the actual PV power in the peak zone $y_{j, d}$.

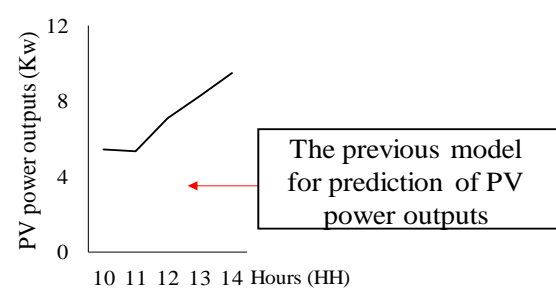

(a) With future meteorological information
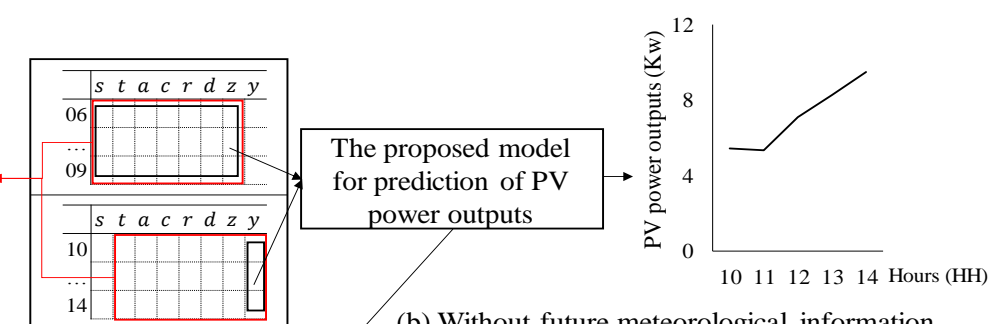

(b) Without future meteorological information

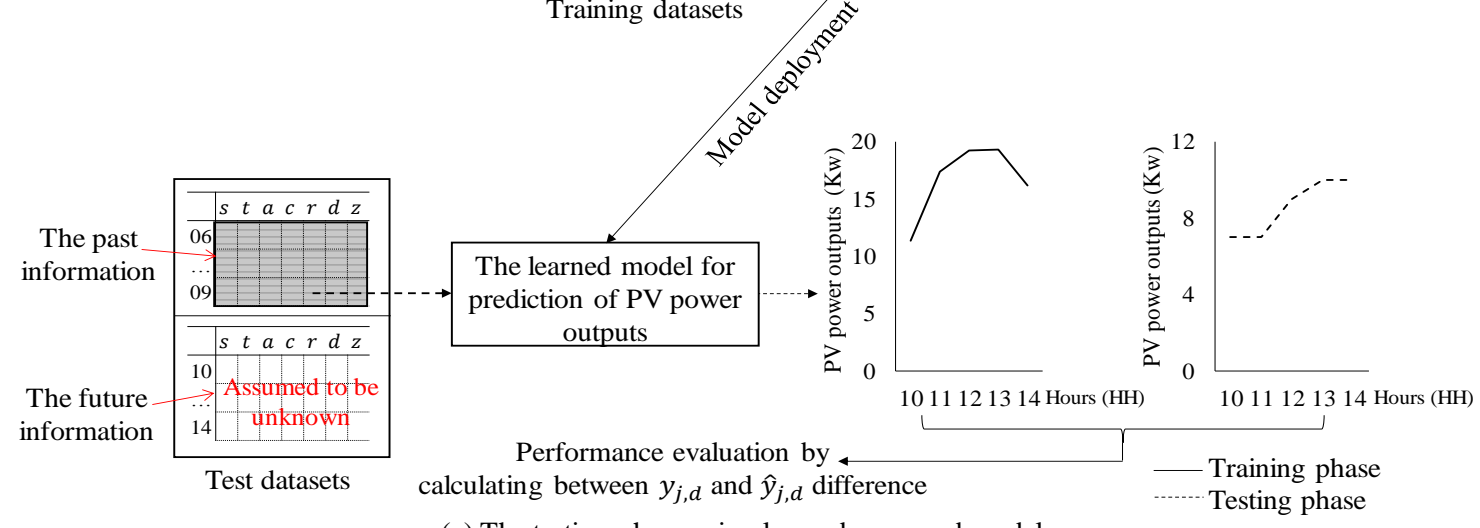

(c) The testing phase using learned proposed model

Figure 2: a Proposed framework based on a neural network for PV power outputs in the peak zone

Specifically, in the learning phase, the weights of the proposed framework model were updated towards minimizing the errors between the $\hat{y}_{j, d}$ and $y_{j, d}$. The proposed framework model consists of an input layer with $n$ nodes where each node is for a value, one or more recurrent hidden layers whose details vary according to the prediction model, and an output layer with $m$ nodes, where each node is for the prediction of PV power output in the peak zone.

In the testing phase, we use the learned weights from the learning phase. As mentioned previously, our framework model estimates the PV power output in the peak zone for 5 hours in a day by computing between the weights and against invisible input values that consisted of the preceding information for 4 hours. The performance of the proposed framework model was evaluated by calculating the difference between the $\hat{y}_{j, d}$ and $y_{j, d}$. Therefore, since the output timescales were more than the input timescales, the prediction of PV power outputs in the peak zone was a more crucially difficult problem.

To improve the prediction of the PV power outputs in a peak zone, the proposed models have network structures such as input, hidden, and output layers, as shown in Figure 3. The check box on the recurrent hidden layer represents the cell, it determines how much short term and long term information regarding relationships between the PV power outputs in the peak zone and the preceding information to store. Since taking advantage of multilayer RNNs enables the use of more layers to capture long-term dependencies of the input sequence values (Zhang et. al., 2017), we set the number of recurrent hidden layers to two.

The input layer takes $x_{k, d}$ and the upper recurrent hidden layer takes output $h_{k, d}$ from the lower input layer as the input $x_{k, d}$. The output layer was located on the cross of the recurrent network hidden layer. The activation and loss functions used were the ReLU function and MSE function, respectively. Although there are many learning algorithms for RNN based models, we applied 
the most known and used backpropagation through time method with the Adagrad optimizer. Therefore, the output value was estimated as the predicted PV power output in the peak zone by calculating the errors between the $\hat{y}_{j, d}$ and $y_{j, d}$ using the MSE function, and the connection weights determined using the optimization method changed depending on the error values.

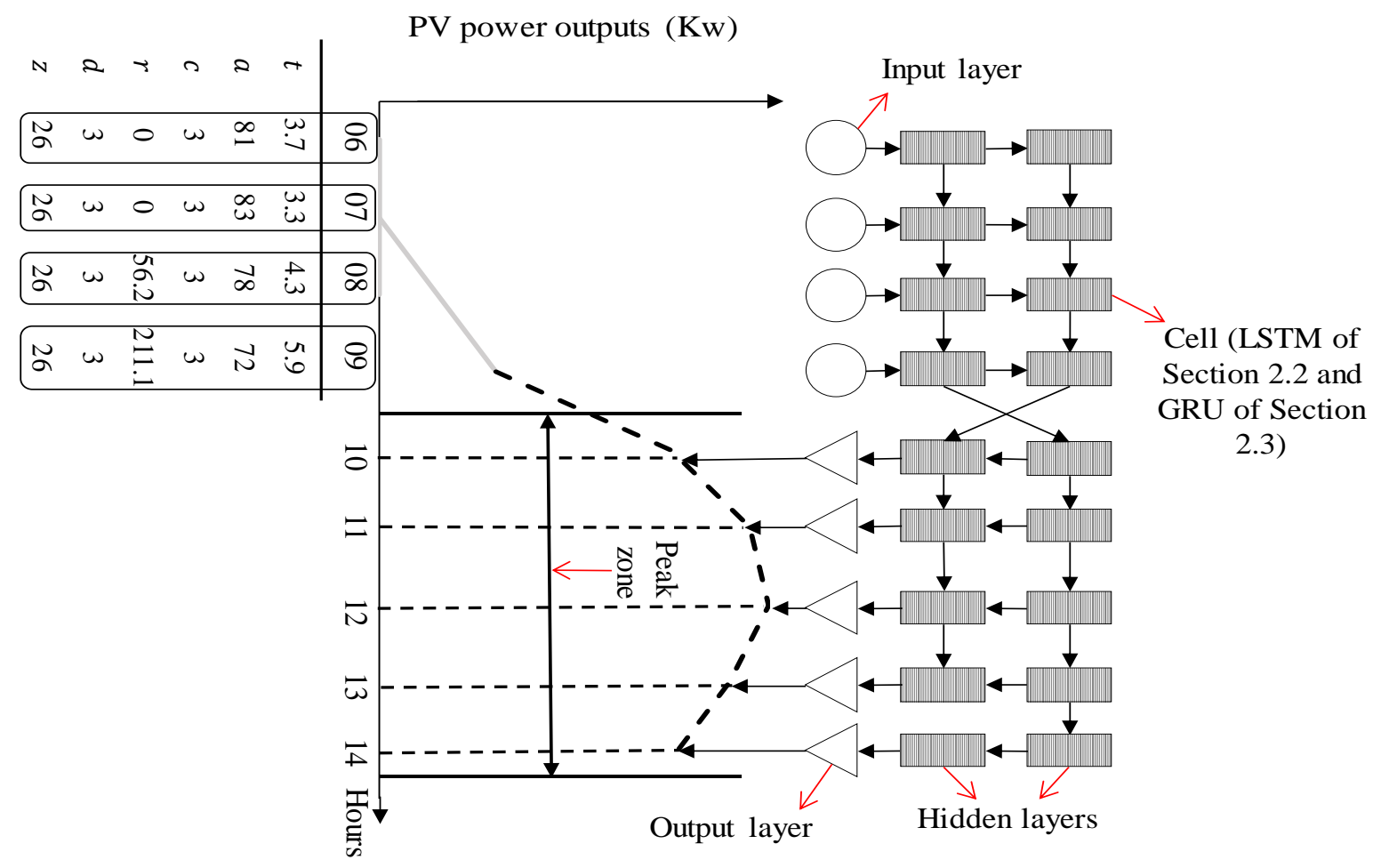

Figure 3: Network structure of the proposed recurrent neural network-based models

To precisely comprehend sequential hidden pattern correlations between PV power outputs in the peak zone and the preceding information in a day and across days, the proposed LSTM based model was adopted. This model is widely applied to solve sequence data problems such as vision tasks and language translation. The proposed LSTM based model has a cell to control the amount of short term and long term memory for accurate prediction of the PV power outputs in the peak zone. Therefore, this model dependently learns to search for a better solution at each time step for the accurate prediction of PV power outputs in the peak zone.

\section{EXPERIMENT RESULTS}

For performance evaluation using proposed models in this paper, we used a PV power output dataset spanning 39 months (from June 1, 2013, to August 31, 2016) from a PV operation located in Gumi city in South Korea. The meteorological information related to the area where the PV operation was located was obtained from the open datasets provided by the Korea government. The number of instances was 16,820, among them, 11,930 instances on June 01, 2013 to September 30, 2015 were used to train the models for the prediction of the PV power outputs in the peak zone.

The remaining 3,196 and 1,694 instances were used as invisible data for the validating and testing performance evaluations. The input values used were information prior to the peak zone, such as the PV power output, meteorological information, and seasonal information, and only output values used were the PV power output in the peak zone. The temperature and humidity values have units of Celsius and percent, respectively, and the ranges of the humidity and cloudiness values were 0 to 100 and 0 to 10 , respectively, with higher values indicating more wetness and clouds in the air.

To evaluate the performances of the proposed models, we calculated the root mean square error (RMSE) and mean absolute error $(M A E)$ based on the actual and the predicted PV power output in the peak zone for a given input instance. As shown in Figure 4, the loss and validation values of the proposed models decreased as the number of epochs increased. These results demonstrate that the proposed models successfully learn by attempting to find better solutions to understand the given training datasets. 


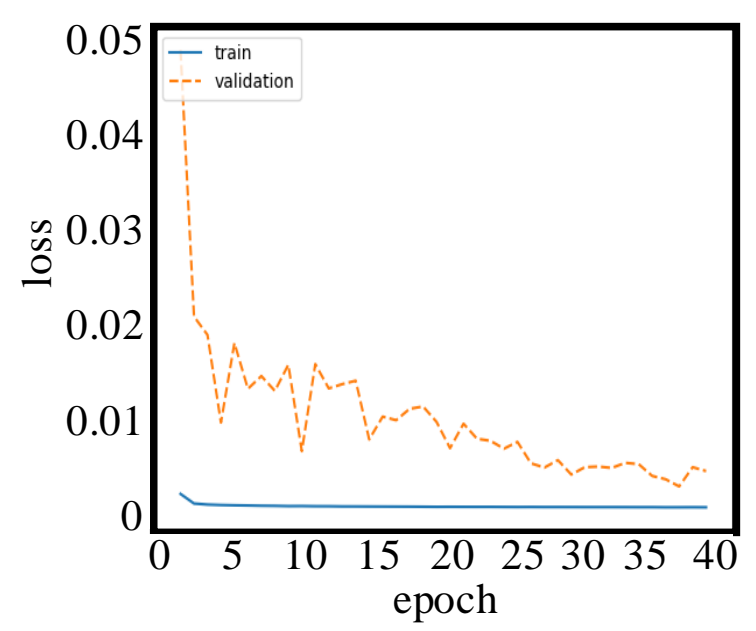

Figure 4: Changes of train and validation loss values according to the number of epochs

Conventional models underperform compared to the proposed models as shown in Figure 5. In particular, ANN-based model shows quite less performance both in terms of MAE and RMSE values. In terms of MAE value, the proposed LSTM based model has improved performance by $29 \%$ and $18 \%$, respectively, compared to ANN and DNN based models, and in terms of RMSE value, grow by $57 \%$ and $40 \%$, respectively.
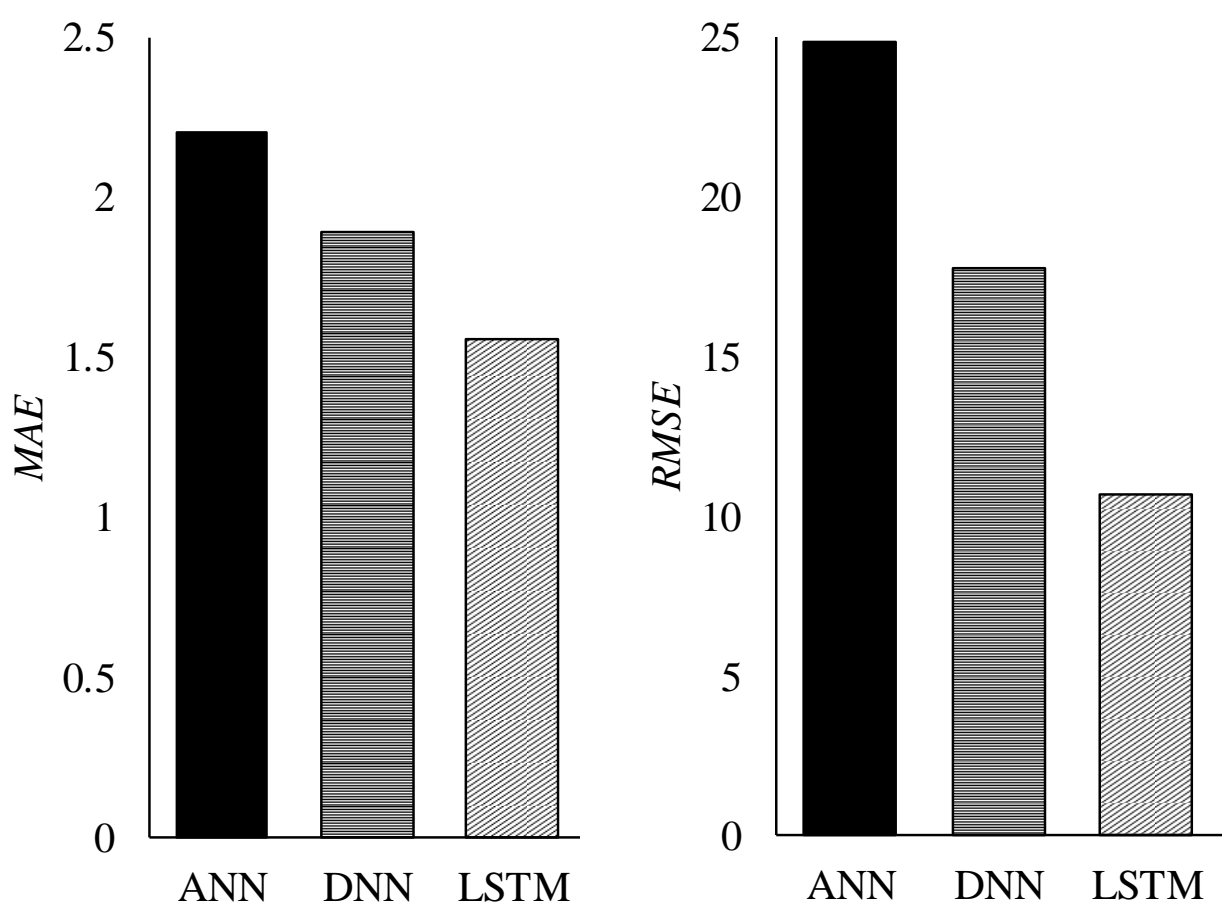

Figure 5: Performance comparisons

\section{CONCLUSIONS}

In this paper, we propose the PV power prediction in the peak zone methods based on recurrent neural networks without future meteorological information, unlike the previous approaches. First, we evaluate the performance of the proposed models compared to conventional models, which ANN and DNN based models, by calculating errors between predicted and actual PV power outputs in the peak zone using real-world datasets. Next, in two aspects of short term and long term, the proposed and conventional models estimate the PV power in the peak zone. ANN-based model underperforms for most of the experiment settings. On the other hand, recurrent neural network-based models show quite successful performances. 
This work was supported by the National Research Foundation of Korea (NRF) grants funded by the Korea government (MSIP) (NRF-2017R1D1A1B03035639).

\section{REFERENCES}

[1] Liu, G.; Xu, Y.; Tomsovic, K. Bidding strategy for microgrid in day-ahead market based on hybrid stochastic/robust optimization. IEEE Trans. Smart Grid 2016, 7, 227-237.

[2] Zhou, Y.; Wang, C.; Wu, J.; Wang, J.; Cheng, M.; Li, G. Optimal scheduling of aggregated thermostatically controlled loads with renewable generation in the intraday electricity market. Appl. Energy 2017, doi:10.1016/j.apenergy.2016.12.008.

[3] Chong, E.; Han, C.; Park, F.C. Deep learning networks for stock market analysis and prediction: Methodology, data representations, and case studies. Expert Syst. Appl. 2017, doi:10.1016/j.eswa.2017.04.030.

[4] Sensfuß, F.; Ragwitz, M.; Genoese, M. The merit-order effect: A detailed analysis of the price effect of renewable electricity generation on spot market prices in Germany. Energy Policy 2008, doi:10.1016/j.enpol.2008.03.035.

[5] Jiahao, K.; Jun, L.; Qifan, L.; Wanliang, F.; Zhenhuan, C.; Linlin, L.; Tieying, G. Photovoltaic power forecasting based on artificial neural network and meteorological data. In TENCON 2013 - 2013 IEEE Region 10 Conference (31194); 2013; pp. 1-4.

[6] Ashraf, I.; Chandra, a Artificial neural network based models for forecasting electricity generation of grid connected solar PV power plant. Int. J. Glob. Energy Issues 2004, 21, 119-130.

[7] Mellit, A.; Shaari, S. Recurrent neural network-based forecasting of the daily electricity generation of a Photovoltaic power system. Ecol. Veh. Renew. Energy (EVER), Monaco, March 2009, 26-29.

[8] Chen, C.; Duan, S.; Cai, T.; Liu, B. Online 24-h solar power forecasting based on weather type classification using artificial neural network. Sol. Energy 2011, 85, 2856-2870, doi:10.1016/j.solener.2011.08.027.

[9] Zhang, S.; Liu, X.; Xiao, J. On geometric features for skeleton-based action recognition using multilayer LSTM networks. In Proceedings - 2017 IEEE Winter Conference on Applications of Computer Vision, WACV 2017 ; 2017. 